\title{
The potential mechanism of the detrimental effect of defibrillation prior to cardiopulmonary resuscitation in prolonged cardiac arrest model
}

\author{
Joon-Ho Bae', Chan-Woo Park', Jun-Hwi Cho',*, Yoon-Sung Kim', Hui-Young Lee², Moo-Ho Won ${ }^{3}$ \\ ${ }^{1}$ Department of Emergency Medicine and Institute of Clinical Science, Kangwon National University Hospital, \\ School of Medicine, Kangwon National University, Chuncheon, Korea \\ ${ }^{2}$ Department of Internal Medicine, Kangwon National University Hospital, School of Medicine, \\ Kangwon National University, Chuncheon, Korea \\ ${ }^{3}$ Departmemt of Neurobiology, School of Medicine, Kangwon National University, Chuncheon, Korea
}

\begin{abstract}
Defibrillation is no longer universally recommended as initial intervention for the reversal of ventricular fibrillation (VF) after a prolonged and untreated cardiac arrest. We sought to examine this issue in an animal model where a prolonged untreated VF was induced. The aim of this study was to investigate the potential mechanism of the detrimental effect of defibrillation prior to cardiopulmonary resuscitation (CPR) in prolonged cardiac arrest model. VF was electrically induced in 32 domestic male swine weighing $40 \pm 3 \mathrm{~kg}$ and remained untreated for 15 minutes. The animals were then randomly allocated to either the initial defibrillation group or the chest compression group. Mean aortic pressure, right atrial pressure and coronary perfusion pressure (CPP) were continuously measured during the performance. The dimensions of the left ventricle (LV) were assessed by echocardiographic methods. The CPP induced by CPR after defibrillation was significantly lower in the initial defibrillation group than in the chest compression group; 1 minute after defibrillation $(9 \pm 3 \mathrm{mmHg}$ vs. $14.8 \pm 7 \mathrm{mmHg}(P<0.05))$, and after 5 minutes $16 \pm 5$ $\mathrm{mmHg}$ vs. $21.7 \pm 1 \mathrm{mmHg}(P<0.05)$. The $\mathrm{LV}$ volumes were reduced from $18 \pm 2 \mathrm{mmHg}$ to $14 \pm 1 \mathrm{mmHg}$ after defibrillation $(P<\mathbf{0 . 0 5})$. In brief, this study showed that the conducting defibrillation prior to chest compression may cause a contracture of the LV, resulting in lowering CPP, thus dropping the efficiency of chest compression in a prolonged cardiac arrest model.
\end{abstract}

Key words: Defibrillation, ventricular fibrillation, contracture, left ventricle

Received 27 March 2014; Revised version received 23 May 2014; Accepted 24 May 2014

Out-of-hospital cardiac arrest due to ventricular fibrillation (VF) or pulseless ventricular tachycardia (VT) associated with good outcomes including high rate of survival [1]. Immediate defibrillation has traditionally been considered to be the priority in out-of-hospital resuscitation algorithms, taking precedence over the initiation of cardiopulmonary resuscitation (CPR) when the cardiac arrest is due a shockable rhythm such as VF/ VT.

Cardiopulmonary resuscitation is only method of treatment in patients with sudden cardiac death. The chain of survival consist of early access, early CPR, early defibrillation, early advanced cardiac life support and post-resuscitation care during treatment of cardiac arrest [2].

Defibrillation is a very important treatment for patients with VF. However, immediate defibrillation is not recommendable in case of a prolonged cardiac arrest due to VF [2]. It is a current trend to conduct CPR prior to the attempt of defibrillation, which results in a better survival rate [1]. Nevertheless, there are some studies that reported opposite research results and immediate

*Corresponding author: Jun-Hwi Cho, Department of Emergency Medicine, School of Medicine, Kangwon National University, Chuncheon 200-701, Korea

Tel: +82-33-258-2378; Fax: +82-33-258-2451; E-mail: cjhemd@kangwon.ac.kr

This is an Open Access article distributed under the terms of the Creative Commons Attribution Non-Commercial License (http://creativecommons.org/licenses/ by-nc/3.0) which permits unrestricted non-commercial use, distribution, and reproduction in any medium, provided the original work is properly cited. 
defibrillation is still advocated sometimes [2,3].

In 2005 the American Heart Association released resuscitation guidelines that included major changes to cardiac arrest management including a statement supporting delaying initial defibrillation to provide a brief period of effective CPR in patients with unwitnessed out-of-hospital cardiac arrest due to VF [2].

We observed in a prolonged cardiac arrest model that showed that pigs after received defibrillation had difficulties to be resuscitated. We hypothesized that defibrillation without chest compression may cause left ventricular (LV) contracture in the prolonged cardiac arrest model that leads to poorer return of spontaneous circulation (ROSC) in the initial defibrillation group than in the chest compression group in our study.

The aim of this study was to investigate the potential mechanism of the detrimental effect of defibrillation prior to CPR in prolonged cardiac arrest model.

\section{Materials and Methods}

\section{Methods}

The experiments were performed in an established swine model of cardiac arrest. All animals $(n=32)$ received humane care in compliance with the "Principles of Laboratory Animal Care" formulated by the National Society for Medical Research and the Guide for the Care and Use of Laboratory Animals prepared by the Institute of Laboratory Animal Resources and published by the National Institutes of Health (NIH publication 86-32, revised 1985). This experiment was carried out when I visited Weil Institute of Critical Care Medicine and the Institutional Animal Care and Use Committee of the Weil Institute of Critical Care Medicine approved the protocol. The animal laboratories of the Weil Institute are fully accredited by American Association for Accreditation of Laboratory Animal Care (AAALAC) International.

\section{Animal preparation}

Thirty-two male Yorkshire-X domestic swine (Sus scrofa) weighing $40 \pm 3 \mathrm{~kg}$ were used in this study. The animals were fasted overnight except for free access to water. Anesthesia was initiated by intramuscular injection of ketamine $(20 \mathrm{mg} / \mathrm{kg})$ and completed by ear vein injection of sodium pentobarbital $(30 \mathrm{mg} / \mathrm{kg})$. A cuffed endotracheal tube was advanced into the trachea. The animals were mechanically ventilated with a volume- controlled ventilator (Model MA-1, Puritan-Bennett, Carlsbad, CA, USA) with a tidal volume of $15 \mathrm{~mL} / \mathrm{kg}$, and $\mathrm{FiO}_{2}$ of 0.21 . End-tidal $\mathrm{CO}_{2}$ was monitored with an infrared capnometer (Model 01R-7101A, Nihon Kohden Corp, Tokyo, Japan). Respiratory frequency was adjusted to maintain $\mathrm{PetCO}_{2}$ between 35 and $40 \mathrm{mmHg}$. For the measurement of aortic pressure (AOP), a fluid filled catheter was advanced from the right femoral artery into the thoracic aorta. For the measurements of right atrial pressure (RAP) and pulmonary arterial pressure (PAP), a 7-French, thermodilution-tipped catheter was advanced from the right femoral vein and flow-directed into the pulmonary artery. For inducing VF, a 5-French pacing catheter (EP Technologies, Inc., Mountain View, CA, USA) was advanced from the right jugular vein into the right ventricle. The position of catheters was confirmed by characteristic pressure morphology and X-ray. The pacing catheter was removed after onset of VF.

\section{Experimental procedures}

Fifteen minutes before inducing cardiac arrest, baseline measurements were obtained and the animals were then randomized to initial defibrillation or chest compression. VF was induced by $1 \mathrm{~mA}$ alternating current delivered to the right ventricular endocardium through a pacing catheter. Mechanical ventilation was discontinued after onset of VF. Prior to starting the resuscitation procedure, the pacing catheter was withdrawn to avoid injury during chest compression.

In the chest compression group (16 animals, delayed defibrillation), fifteen minutes after onset of VF, chest compression was started with a pneumatic piston-driven chest compressor (Thumper $^{\circledR}$, Model 1000, Michigan Instruments, Grand Rapids, MI, USA). The chest compression was programmed to provide 100 compressions/ minute and synchronized to provide a compression/ ventilation ratio of 30:2 with equal compression-relaxation intervals, i.e. a $50 \%$ duty cycle. The compression depth was adjusted to decrease the anterior-posterior diameter by $25 \%$. Coincident with starting precordial compression, the animals were mechanically ventilated with tidal volume of $15 \mathrm{~mL} / \mathrm{kg}$ and $\mathrm{FiO}_{2}$ of 1.0. After 2 minutes of chest compression, one dose of epinephrine $(30 \mu \mathrm{g} / \mathrm{kg})$ was injected into the right atrium. After another 3 minutes of chest compression, one $120 \mathrm{~J}$ biphasic electrical defibrillation was delivered between the right infra-clavicular electrode and the apical electrode with a Heartstart $^{\circledR}$ XL defibrillator (Philips Medical Systems, 
Experimental Procedures

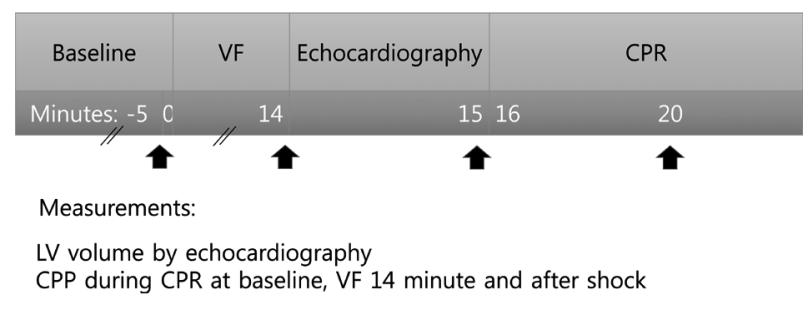

Figure 1. Experimental process. Baseline data acquisition were completed before induced VF. LV volume was measured at 14 minutes after VF. CPR with chest compression and ventilation begun at 15 minutes after VF. VF: ventricular fibrillation, LV: left ventricle, CPP: coronary perfusion pressure, CPR: cardiopulmonary resuscitation.

Andover, MA, USA). If an organized cardiac rhythm with mean aortic pressure (MAP) of more than 60 $\mathrm{mmHg}$ persisted for an interval of 5 minutes or more, the animal was regarded as successfully resuscitated. If the ROSC was not achieved, CPR was resumed for 1 minute prior to the next defibrillation attempt. This sequence was repeated until the animal was either successfully resuscitated or pronounced dead after a total of 15 minutes of CPR. Other doses of epinephrine were injected separately at the 7th and 12th minute following the initiation of CPR.

In the initial defibrillation group (16 animals, immediate defibrillation), fifteen minutes after onset of VF, immediate defibrillation was delivered. The chest compression was started after defibrillation. The defibrillation was done with biphasic $120 \mathrm{~J}$. CPR was conducted with the same method as the chest compression group (Figure 1).

Echocardiograms were obtained immediately before starting chest compression in both groups. Dimensions of LV were assessed by echocardiographic methods in parasternal short axis view. The degree of LV contracture was calculated by comparison with volume of the LV before and after defibrillation (Figure 2).

\section{Statistical analyses}

Continuous variables were presented as mean $\pm \mathrm{SD}$. Student's t-test was used to compare continuous variables between two groups. Categorical variables are reported as counts and percentages. Primary and secondary outcomes were binary, and the chi-square test or Fisher's exact test, as appropriate, was used to compare outcomes in chest compression first and initial defibrillation groups. A value of $P<0.05$ was considered significant. Analyses were carried out using SPSS V.11.0 software (IBM Corp., Chicago, IL, USA).

\section{Results}

In the present study, we examined the potential mechanism of the detrimental effect of defibrillation prior to CPR in prolonged cardiac arrest model. The demographic data showed no differences between the chest compression group and the initial defibrillation group (Table 1). Nine of $16(56.3 \%)$ animals treated with chest compression prior to defibrillation achieved ROSC, while no animals without chest compression had ROSC $(P<0.01)$. The CPP induced by CPR in the initial defibrillation group after defibrillation was significantly decreased compared with that in the chest compression group; 1 minute after chest compression $(9 \pm 3 \mathrm{mmHg}$ vs. $14.8 \pm 7 \mathrm{mmHg}(P<0.05)$, and after 5 minutes $16 \pm 5$ $\mathrm{mmHg}$ vs $21.7 \pm 1 \mathrm{mmHg}(P<0.05)$ (Figure 3$)$. In addition, the $\mathrm{LV}$ volumes were decreased from $18 \pm 2 \mathrm{mmHg}$ to $14 \pm 1 \mathrm{mmHg}$ after defibrillation $(P<0.05)$ (Figure 4$)$.

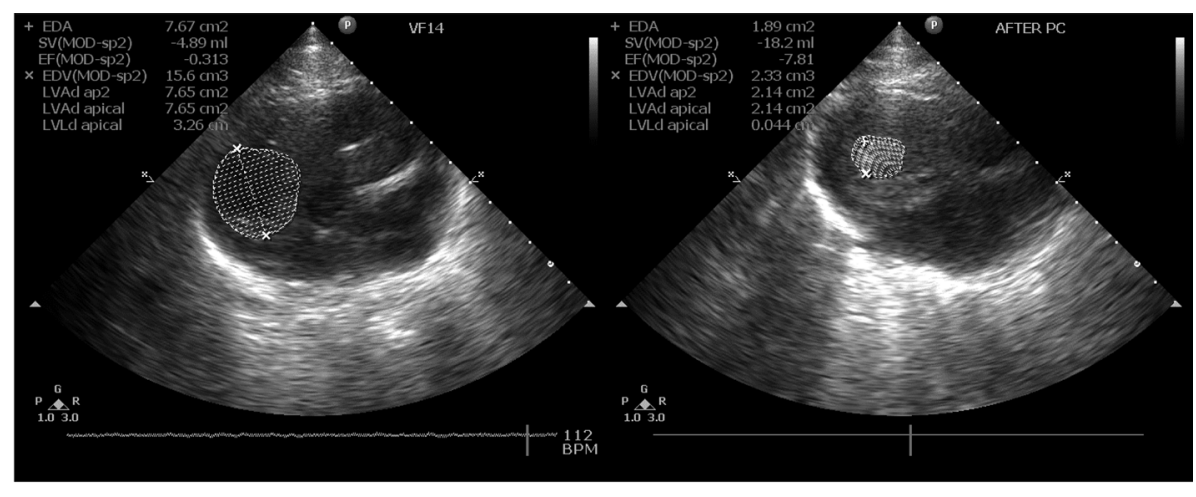

14 minutes after induced VF

After defibrillation

Figure 2. The echocardiographic methods of measurement of $L V$ volume. This figure shows $L V$ volume at 14 minutes after induced VF and after defibrillation. VF: ventricular fibrillation, LV: left ventricle. 
Table 1. Demographic data shows no differences between chest compression group and initial defibrillation group

\begin{tabular}{lccc}
\hline & Chest compression group $(\mathrm{n}=16)$ & Initial defibrillation group $(\mathrm{n}=16)$ & $P$-value \\
\hline Body weight, $\mathrm{kg}$ & $41 \pm 2$ & $40 \pm 3$ & 0.460 \\
Heart rate, beat/min & $132 \pm 25$ & $143 \pm 36$ & 0.499 \\
Mean aortic pressure, $\mathrm{mmHg}$ & $112 \pm 16$ & $113 \pm 13$ & 0.837 \\
Mean right atrial pressure, $\mathrm{mmHg}$ & $7 \pm 2$ & $7 \pm 1$ & 0.764 \\
Mean pulmonary artery pressure, $\mathrm{mmHg}$ & $22 \pm 4$ & $24 \pm 6$ & 0.566 \\
Ejection Fraction, \% & $69.8 . \pm 3.3$ & $66.6 \pm 2.8$ & 0.056 \\
Cardiac output, l/min & $7 \pm 1$ & $7 \pm 1$ & 0.995 \\
End-tidal $\mathrm{CO}_{2}$ & $37 \pm 2$ & $36 \pm 2$ & 0.550 \\
\hline
\end{tabular}

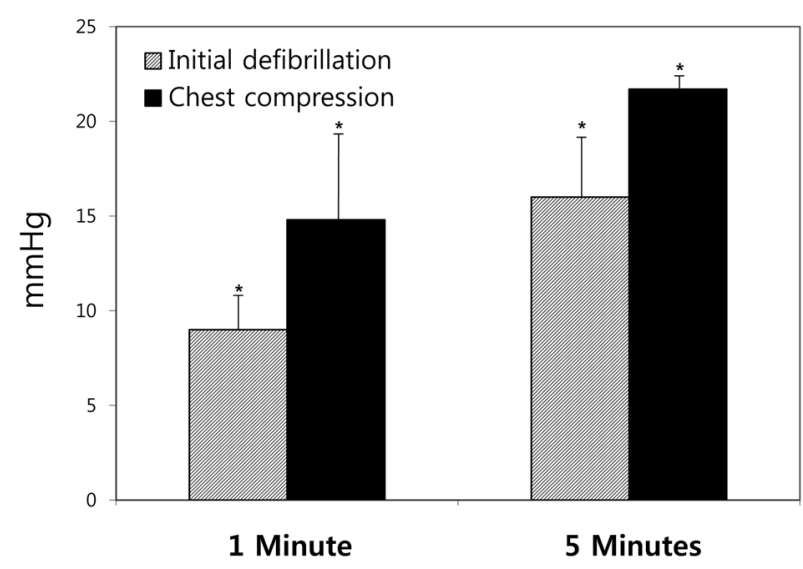

Figure 3. The comparison of coronary perfusion pressure during CPR between two groups. The CPP of the chest compression group was higher than initial defibrillation group in one minute and 5 minutes after chest compression $\left({ }^{*} P<0.05\right.$ : significantly different between two groups). CPR: cardiopulmonary resuscitation, CPP: coronary perfusion pressure.

\section{Discussion}

Most of sudden cardiac arrest patients show cardiac rhythms of ventricular fibrillation when discovered. Defibrillation is the most important treatment for patients who suffer from ventricular fibrillation [2]. The survival of cardiac arrest patients after ventricular fibrillation depends highly on the time span between the onset of ventricular fibrillation and the defibrillation performance. An elapse of one minute from the occurrence of ventricular fibrillation was reported to reduce the success rate of defibrillation by $7-10 \%$ [4].

Recently, however, there have been many studies reporting that the survival rate may increase when $\mathrm{CPR}$ is conducted before defibrillation in case of prolonged ventricular fibrillation [1]. According to the 2005 ILCOR (International Liaison Committee on Resuscitation) guideline, it is required to conduct CPR before defibrillation in case of a not witnessed cardiac arrest due to $\mathrm{VF}$ outside of hospitals [2].

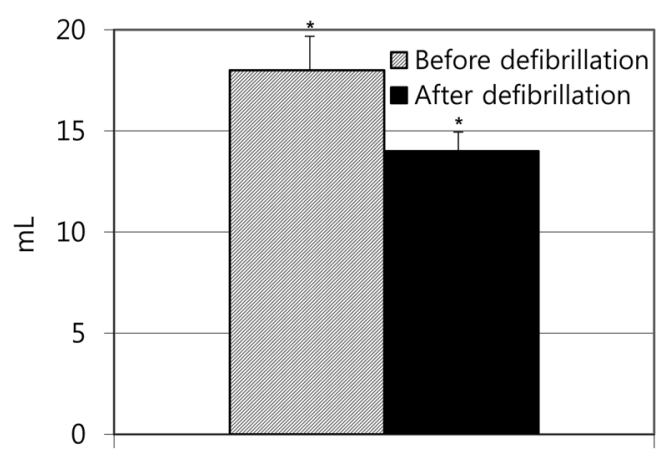

Figure 4. The volume of left ventricle was decreased after defibrillation in the prolonged cardiac arrest model $\left({ }^{\star} P<0.05\right.$ : significantly different between two groups).

The theoretical background for requiring CPR first in case of prolonged VF is that it can restrict the end organ ischemia, lead the cardiac metabolic state to a proper condition for defibrillation and reduce the amount of a reperfusion injury [5-8]. In spite of such theoretical background and several reports with data, there are a number of studies with an opposing view also.

Simpson et al. surveyed 658 cardiac arrest patients outside of the hospital. They proved that there was no difference in the survival rates between the patient groups, where CPR was conducted before defibrillation and where defibrillation was conducted in advance [9]. Baker et al. also advocated an immediate defibrillation, asserting that CPR prior to defibrillation had no gain at all [3]. However, in our swine model study, the rate of ROSC was higher in the group where CPR was conducted before defibrillation than in the group where immediate defibrillation was conducted in case of the prolonged ventricular fibrillation. We found in the prolonged cardiac arrest model that a defibrillation prior to chest compression could worsen a LV contracture. Moreover, a more severe LV contracture was observed in cardiac arrest if continued for 15 minutes. Berg et al. observed that the left ventricular volume decreased as 
the right ventricular volume decreased for one minute at the initial stage of post-VF arrest. Then the left ventricular volume increased slightly as the right ventricular volume slightly decreased for further three minutes and thereafter the left ventricular volume decreased again [10]. Cooley et al. reported a cardiac arrest that had already occurred without proper treatment for 20 to 30 minutes could lead to irreversible damages of the myocardium resulting in a serious contracture named "stone heart" [11].

In this study, defibrillation was conducted 15 minutes after the occurrence of cardiac arrest when the heart reached the condition that the perfusion had dropped enough. The left ventricular volume was found to be decreased after the conduction of defibrillation, indicating that electrical defibrillation itself accelerated the contracture of the LV causing the decreasing efficiency of chest compression.

In our present study, there was no success of ROSC in the animals with initial defibrillation. That may be possibly related with the contracture of the LV. In addition, the difference of coronary perfusion pressure between the chest compression group and the initial defibrillation group can be deemed to arise from the contracture of the LV. Further studies may be required on the cause of LV contracture and its progress during cardiac arrest.

Furthermore, in our cardiac arrest swine model, the rate of ROSC was lower in the initial defibrillation group than in the chest compression group in the prolonged cardiac arrest.

In brief, these observations suggest that conducting defibrillation prior to chest compression may cause a contracture of the $\mathrm{LV}$, resulting in lowering CPP, thus dropping the efficiency of chest compression in a prolonged cardiac arrest model.

\section{References}

1. Cheung W, Flynn M, Thanakrishnan G, Milliss DM, Fugaccia E. Survival after out-of-hospital cardiac arrest in Sydney, Australia. Crit Care Resusc 2006; 8(4): 321-327.

2. AHA. Guidelines for cardiopulmonary resuscitation and emergency cardiovascular care-an international consensus on science. Circulation 2005; 112(suppl.).-1--4.

3. Baker PW, Conway J, Cotton C, Ashby DT, Smyth J, Woodman RJ, Grantham H; Clinical Investigators. Defibrillation or cardiopulmonary resuscitation first for patients with out-ofhospital cardiac arrests found by paramedics to be in ventricular fibrillation? A randomised control trial. Resuscitation 2008; 79(3): 424-431.

4. Hwang SO, Lim KS. Cardiopulmonary Resuscitation and Advanced Cardiovascular Life Support. 3rd ed. Seoul, Koonja; 2006; pp 56.

5. Chandra NC. Mechanisms of blood flow during CPR. Ann Emerg Med 1993; 22(2 Pt 2): 281-288.

6. Steen S, Liao Q, Pierre L, Paskevicius A, Sjöberg T. The critical importance of minimal delay between chest compressions and subsequent defibrillation: a haemodynamic explanation. Resuscitation 2003; 58(3): 249-258.

7. Rea TD, Cook AJ, Hallstrom A. CPR during ischemia and reperfusion: a model for survival benefits. Resuscitation 2008; 77(1): 6-9.

8. Chamberlain D, Frenneaux M, Fletcher D. The primacy of basics in advanced life support. Curr Opin Crit Care 2009; 15(3): 198202.

9. Simpson PM, Goodger MS, Bendall JC. Delayed versus immediate defibrillation for out-of-hospital cardiac arrest due to ventricular fibrillation: A systematic review and meta-analysis of randomised controlled trials. Resuscitation 2010; 81(8): 925-931.

10. Berg RA, Sorrell VL, Kern KB, Hilwig RW, Altbach MI, Hayes MM, Bates KA, Ewy GA. Magnetic resonance imaging during untreated ventricular fibrillation reveals prompt right ventricular overdistention without left ventricular volume loss. Circulation 2005; 111(9): 1136-1140.

11. Cooley DA, Reul GJ, Wukasch DC. Ischemic contracture of the heart: "stone heart". Am J Cardiol 1972; 29(4): 575-577. 